\title{
Searches for supersymmetry with the CMS experiment
}

\author{
Alexis Kalogeropoulos ${ }^{1}$ \\ On behalf of the CMS Collaboration \\ ${ }^{1}$ Princeton University
}

\begin{abstract}
We report on recent results by the CMS Collaboration on searches for supersymmetry based on a sample of proton-proton collision data at $\sqrt{s}=13 \mathrm{TeV}$, corresponding to a total integrated luminosity of $35.9 \mathrm{fb}^{-1}$.
\end{abstract}

\section{Introduction}

Supersymmetry (SUSY) [1] is a well-motivated theory beyond the standard model (SM) which provides solutions to several open questions in particle physics, e.g. the cancellation of quadratically divergent loop corrections to the mass of the Higgs boson. In R-Parity [2] conserving SUSY, the lightest SUSY particle (LSP) is stable and can be a viable dark matter candidate. In this talk we review recent results published by the CMS Collaboration [3] based on the Run II dataset at $\sqrt{s}=13$ $\mathrm{TeV}$, with results interpreted in the context of Simplified Models (SMS) [4]-[8], while the full suite of available results can be also found in the CMS SUSY public results page [9].

\section{Strong production}

\subsection{Search for SUSY in multijets and $H_{T}^{\text {miss }}$ (SUS-16-033) [10]}

Searches for strong SUSY production benefit from the higher production cross section compared to other production mechanisms of SUSY. Further, a wide range of experimental signatures and variables are covered, exploiting the fact that depending on the decay mode of the gluinos, mutlijet and b-tagged jet as well as large transverse missing energy (MET) characterize the final state. This analysis uses mutlijets, MET and $\mathrm{H}_{\mathrm{T}}^{\mathrm{miss}}$ (the magnitude of the negative of the vector $\mathrm{P}_{\mathrm{T}}$ sum of the jets of the event) as experimental signature. The baseline selection comprises es at least 2 jets, the $\mathrm{H}_{\mathrm{T}}$ and the $\mathrm{H}_{\mathrm{T}}^{\text {miss }}$ must be higher than $300 \mathrm{GeV}$, while a veto on events with isolated leptons with $\mathrm{P}_{\mathrm{T}}>10 \mathrm{GeV}$ is posed. Further, in order to suppress multijet background (QCD) a cut on the $\Delta \Phi$ between the $\mathrm{H}_{\mathrm{T}}^{\text {miss }}$ and each of the jets is applied and has to be above 0.5 radians. Several search bins are formed in the multidimensional space of $\mathrm{H}_{\mathrm{T}}, \mathrm{H}_{\mathrm{T}}^{\text {miss }}, \mathrm{N}_{\text {jets }}$ and $\mathrm{N}_{\mathrm{b}-\text { jets }}$ as shown in Fig. 1. Regions marked with $\mathrm{C} 1 / \mathrm{C} 2 / \mathrm{C} 3$ are used to estimate the multijet background contribution. 


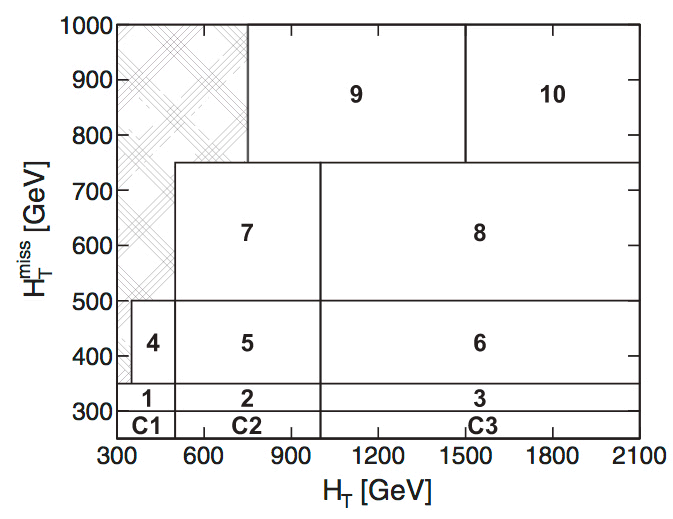

Figure 1: Illustration of the 10 kinematic search intervals in the $\mathrm{H}_{\mathrm{T}}^{\text {miss }}$ versus $\mathrm{H}_{\mathrm{T}}$ plane. Intervals 1 and 4 are discarded for $\mathrm{N}_{\text {jets }}>7$. The intervals labeled $\mathrm{C} 1, \mathrm{C} 2$, and $\mathrm{C} 3$ are control regions used to evaluate the QCD background.

\section{Background estimation}

The evaluation of background is estimated from data in control regions (CR). The background from top quark, $\mathrm{W}+\mathrm{Jets}$ and single top quark stems from leptonic decays of the $\mathrm{W}$ bosons and constitutes the "lost lepton" category, when the lepton lies out of acceptance or it is mis-reconstructed or it is not isolated, or when the lepton is a hadronically decaying $\tau$ lepton (denoted by $\tau_{h}$ ). This contribution is estimated from 1- $\ell$ CRs formed by inverting the $\ell$ vetoes and requiring $\mathrm{M}_{\mathrm{T}}<100 \mathrm{GeV}$. Next, each event in this CR is weighted with a probability to appear in the corresponding $\mathrm{H}_{\mathrm{T}} / \mathrm{H}_{\mathrm{T}}^{\mathrm{miss}} / \mathrm{N}_{\mathrm{jets}} /$ $\mathrm{N}_{\mathrm{b}-\text { jets }}$ bins. In order to evaluate the background due to the $\tau_{h}$, a CR is used with exactly one isolated muon and the similarities between the the $\mu+$ jets and $\tau_{h}+$ jets are exploited; to this end, each $\mu$ is smeared through random sampling of $\tau_{h}$ response function derived from simulation. The response functions express the expected visible- $\mathrm{P}_{\mathrm{T}}$ distribution of a $\tau_{h}$ candidate as a function of the true $\tau$ lepton $\mathrm{P}_{\mathrm{T}}$, taken to be the measured muon $\mathrm{P}_{\mathrm{T}}$ in the CR event. Next, the $\mathrm{H}_{\mathrm{T}}, \mathrm{H}_{\mathrm{T}}^{\text {miss }}, \mathrm{N}_{\text {jets }}$ and $\mathrm{N}_{\mathrm{b} \text {-jets }}$ are calculated for the CR event. Further, the contribution from $Z \rightarrow v \bar{v}$ events is obtained from CRs enriched in $\gamma$ and $\ell^{+} \ell^{-}$inside the Z-mass window requiring also $\mathrm{P}_{\mathrm{T}(\ell \ell)}>200 \mathrm{GeV}$, while in the next step the $\gamma$ and the $\ell \ell$ pair are removed from the event. Finally the $Z \rightarrow v \bar{v}$ is estimated as a function of the fraction of prompt $\gamma$, the fraction of prompt $\gamma$ that are also direct (ie when $\gamma$ is produced via Compton scattering $(q g \rightarrow q g)$ or annihilation $(q q \rightarrow \gamma g)$, corrected for the ratio of $Z \rightarrow v \bar{v} / \gamma$ and other corrections obtained from simulation).

\section{Results}

Fig. 2 presents the observed number of events in all of the search regions (174 in total). The data are compatible with the background estimate and no signs for supersymmetry are observed. The interpretation of results is done in the context of several SMS (Fig. 3 ). 


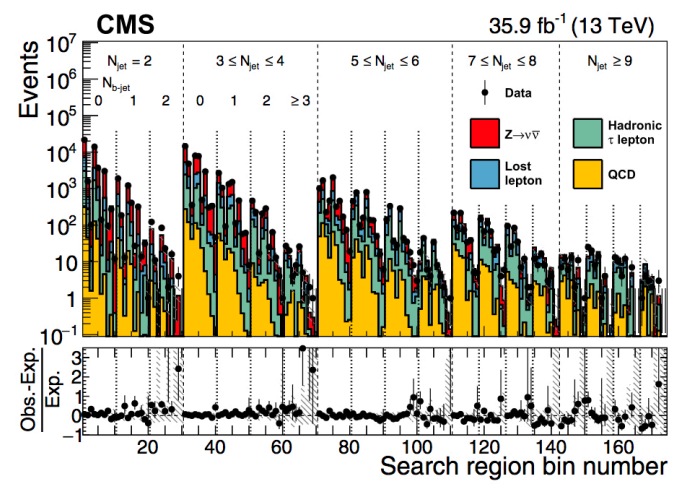

Figure 2: The observed numbers of events and prefit background predictions in the total of 174 search regions of the analysis, ("prefit" means there is no constraint from the likelihood fit.). The hatching indicates the total uncertainty in the background predictions. The lower panel displays the ratio between the data and SM predictions [10].
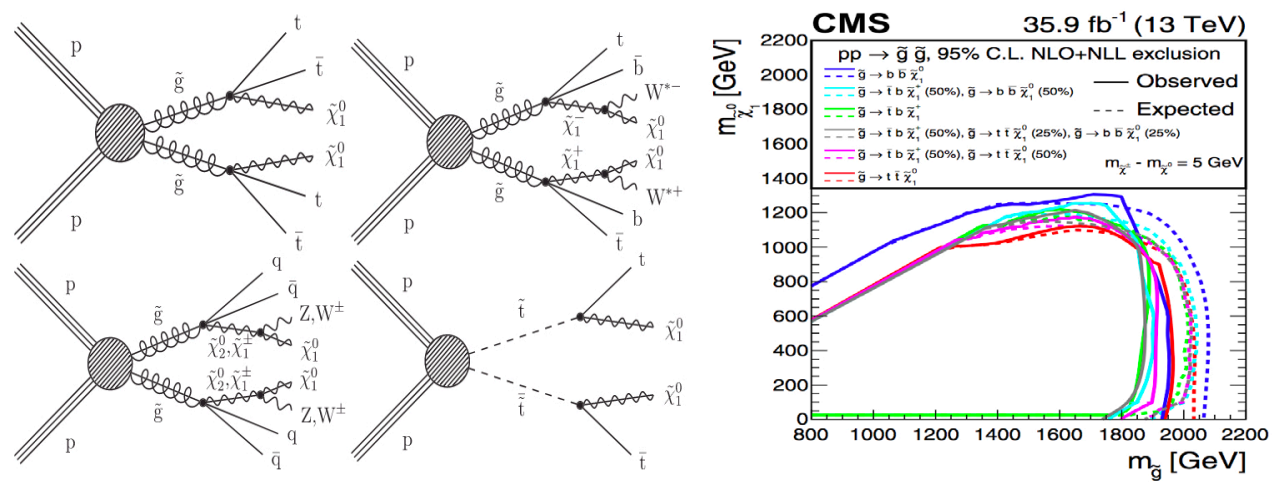

Figure 3: Left: The SMS used for the interpretations of results. Right: The results in the LSP versus gluino plane and for the considered SMS. Roughly, gluino masses up to 2 (1.8) $\mathrm{TeV}$ are excluded for massless (1.2 TeV) LSP at 95\% CL [10].

\section{Third Generation searches}

\subsection{Search for top squark pair with $1-\ell$ in the final state (SUS-16-051) [11]}

The analysis targets the production of top squark pairs in the $1-\ell$ final state. The event selection requires the presence of exactly one lepton, at least two jets, $M_{T}$ higher than $150 \mathrm{GeV}$, and veto on the presence of more leptons or loose tracks. Further, in order to suppress the top quark background contributions to $2-\ell$ final states, a cut on the azimuthal plane between the $\mathrm{P}_{\mathrm{T}}$ and the direction of the closest of the two leading $\mathrm{P}_{\mathrm{T}}$ jets in the event greater than 0.8 radians is posed. The search regions are formed based on the $\mathrm{P}_{\mathrm{T}}$, the $\mathrm{N}_{\text {jets }}$, the MET the $M_{\ell, b}$ as well as the topness variable (which tests for the compatibility with the the $t \bar{t} \rightarrow 2 \ell$ hypothesis when on of the two lepton is lost) with a total of 31 search regions. 


\section{Background estimation}

The background contribution can be categorized in the following main categories :

- Lost-Lepton : events with 2 leptonically decaying W bosons when 1- $\ell$ is lost. Main source is top quark and it is estimated from $2-\ell \mathrm{CR}$.

- 1- $\ell$ bkg : It mainly comes from W boson leptonic decays and small MET. This background can be suppressed with a high MET cut and it is then estimated from CR with no b-jets.

- Finally, the contribution from $Z \rightarrow v \bar{v}$ events with exactly one leptonically decaying $\mathrm{W}$ boson and a $\mathrm{Z}$ boson that decays to a pair of neutrinos, e.g., ttZ+Jets or WZ+Jets. This background is estimated from simulation normalised to data from a dedicated CR.

\section{Results}

With no significant excess over the bkacground only hypotheses, results are interpreted in the context of SMS of top squark pair production with each top squark decaying to a top quark and a neutralino with a branching ratio of $100 \%$ (Fig. 4). The largest uncertainties on the results are due to the limited size of the simulated signal samples, the $b$ tagging efficiency, and the jet energy scale as well. Top squark masses up to $1120 \mathrm{GeV}$ for a massless LSP and LSP masses up to $515 \mathrm{GeV}$ for a $950 \mathrm{GeV}$ top squark mass are excluded. Finally, the white band corresponds to the region where the selection efficiency of top squark events vastly changes and the details of the model and the simulation affect the signal efficiency. No cross section limit is established in that region as well.
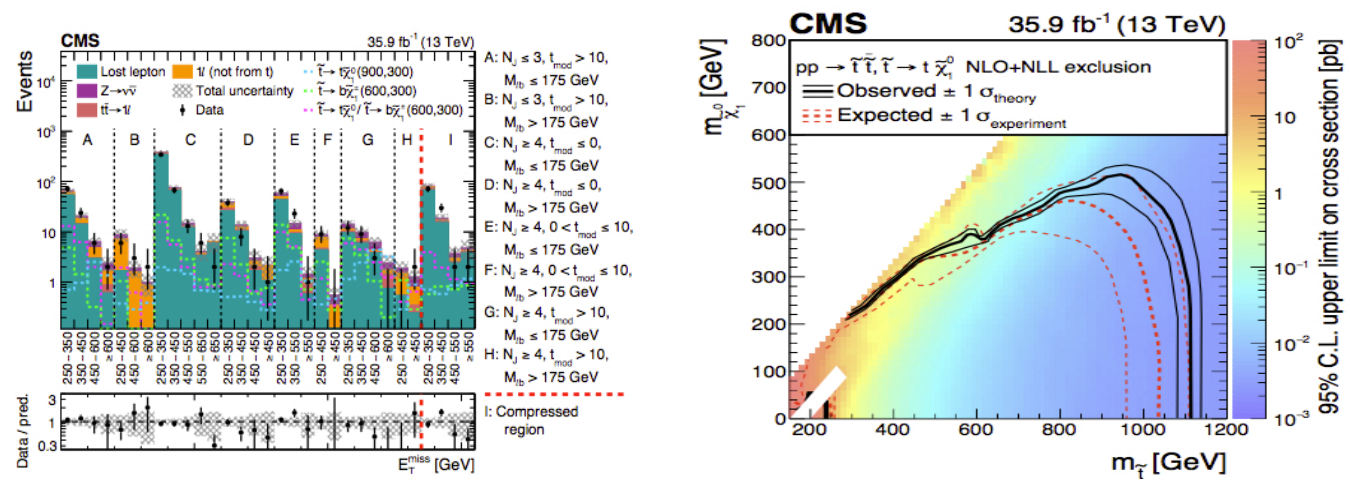

Figure 4: Left : Observed yields compared with the SM prediction for the search regions. The total uncertainty in the background estimate is shown as a shaded band. The expectations for three signal hypotheses are overlaid. Numbers in parentheses in the legend refer to the masses in $\mathrm{GeV}$ of the top squark and the neutralino. Right : The exclusion limits at 95\% CL for direct top squark pair production with decay to a top quark and a neutralino each. The interpretation is done in the stop quark vs. neutralino mass plane [11]. 


\section{Electroweak production}

\subsection{Search for electroweak production of charginos and neutralinos in multilepton final states (SUS-16-039) [12]}

This analysis targets direct electroweak production of charginos and neutralinos in signatures with either two or more leptons (e or $\mu$ ) of the same electric charge, or with three or more leptons, which can include up to two hadronically decaying $\tau$ leptons. In the case of $\tilde{\chi}^{ \pm} \tilde{\chi}_{2}^{0}$ production, they are assumed to be mass-degenerate and wino-like (i.e. superpartners of the SU(2)L gauge fields), and the $\tilde{\chi}_{1}^{0}$ is set to be bino-like, meaning a superpartner of the $\mathrm{U}(1) \mathrm{Y}$ gauge field. The masses of the pure wino-like and bino-like gauginos are governed by two complex gaugino Majorana mass parameters, and can take any value. In this scenario, the $\tilde{\chi}_{1}^{0}$ is the LSP. For the effective $\tilde{\chi}_{1}^{0} \tilde{\chi}_{1}^{0}$ pair production, the $\tilde{\chi}_{2}^{0} \tilde{\chi}^{ \pm}$and $\tilde{\chi}_{1}^{0}$ are assumed to be higgsino-like, i.e. superpartners of the Higgs doublets. In this case, scenarios in which the $\tilde{\chi}_{1}^{0}$ is the next-to-LSP (NLSP) are considered. The search regions are based on the flavour, the multiplicity of leptons or $\tau$ in the final state, the $\mathrm{N}_{\text {jets }}$, the MET, the $\mathrm{M}_{\mathrm{T}}$, the $\mathrm{P}_{\mathrm{T}}$ of the dilepton system as well as the sign (either same-sign or opposite-site).

\section{Background estimation}

The background contributions can be mainly categorized into the following:

- $\mathrm{WZ}$ or $\mathrm{W} \gamma *$ : This background is dominant with 3- $\ell$ in the final state with an opposite-sign-sameflavour (OSSF) pair. This is estimated from a CR with $75<M_{\ell \ell}<105 \mathrm{GeV}, \mathrm{M}_{\mathrm{T}}<100 \mathrm{GeV}$, and $35<$ MET $<100 \mathrm{GeV}$.

- The non-prompt e, $\mu, \tau$ contributions depend on the multiplicity of the leptons and it is dominated by

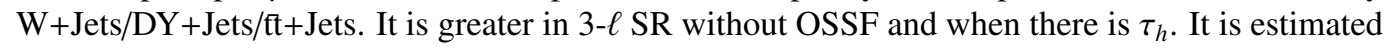
from the "tight-to-loose" ratio in an CR from data.

- Conversion : $\mathrm{W} / \mathrm{Z}$ radiates a $\gamma$ which then converts and this type of background is present in OSSF pair and when there are two same-sign $\ell$. It is estimated in a CR by comparing the $Z \rightarrow \ell \ell$ with $\gamma^{*} \rightarrow \ell \ell$ where one lepton is out of acceptance, and this is compared to predictions from simulation with $\left|\ell-M_{Z}\right|>15 \mathrm{GeV}$.

- Charge mis-iD : This happens when one $\ell$ from OS pair is mis-identified (for example, from bremsstrahlung in tracker). It is relatively small background in same-sign events, and it is estimated by reweighing OS events with mis-identified probability (which is obtained from simulation). In general it is an overall small contribution.

- Rare with multiple $\ell$ : This is the case when same-sign leptons or more than two lepton stem from rare processes (like VVX+Jets, $\mathrm{ttH}+\mathrm{Jets}$ ). This type of background can be suppressed by a b-jet veto and it is estimated from simulation.

\section{Results}

Since no significant deviation with respect to the SM prediction is observed, the results are interpreted in the context of the SMS covering scenarios of $\tilde{\chi}^{ \pm} \tilde{\chi}_{1}^{0}$ production with $\tilde{\ell}_{R}, \tilde{\ell}_{L}$ and $\tilde{\tau}$ mediated decays, decays to 2-body decays to W,Z and models with four higgsinos in the final state, which are illustrated in Fig. 5. Some of the final results are shown in Fig. 6. 

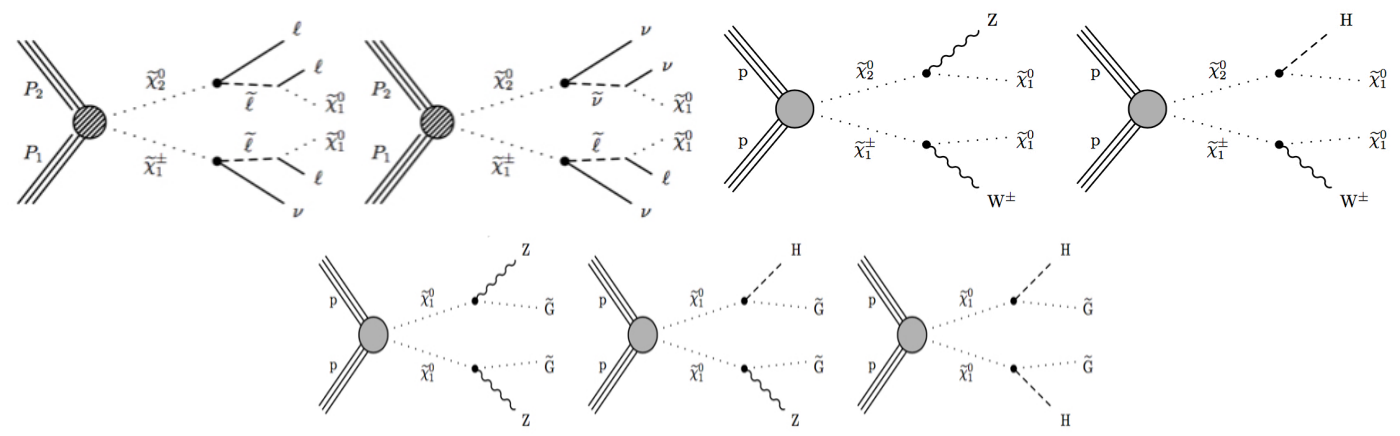

Figure 5: The Feynman diagrams of the SMS models used to interpret the results with $\tilde{\chi}^{ \pm} \tilde{\chi}_{1}^{0}$ with $\tilde{\ell}_{R}$, $\tilde{\ell}_{L}$ and $\tilde{\tau}$ mediated decays (top left), decays to 2-body decays with W,Z and Higgs bosons (top right), and decays with four higgsinos (bottom).
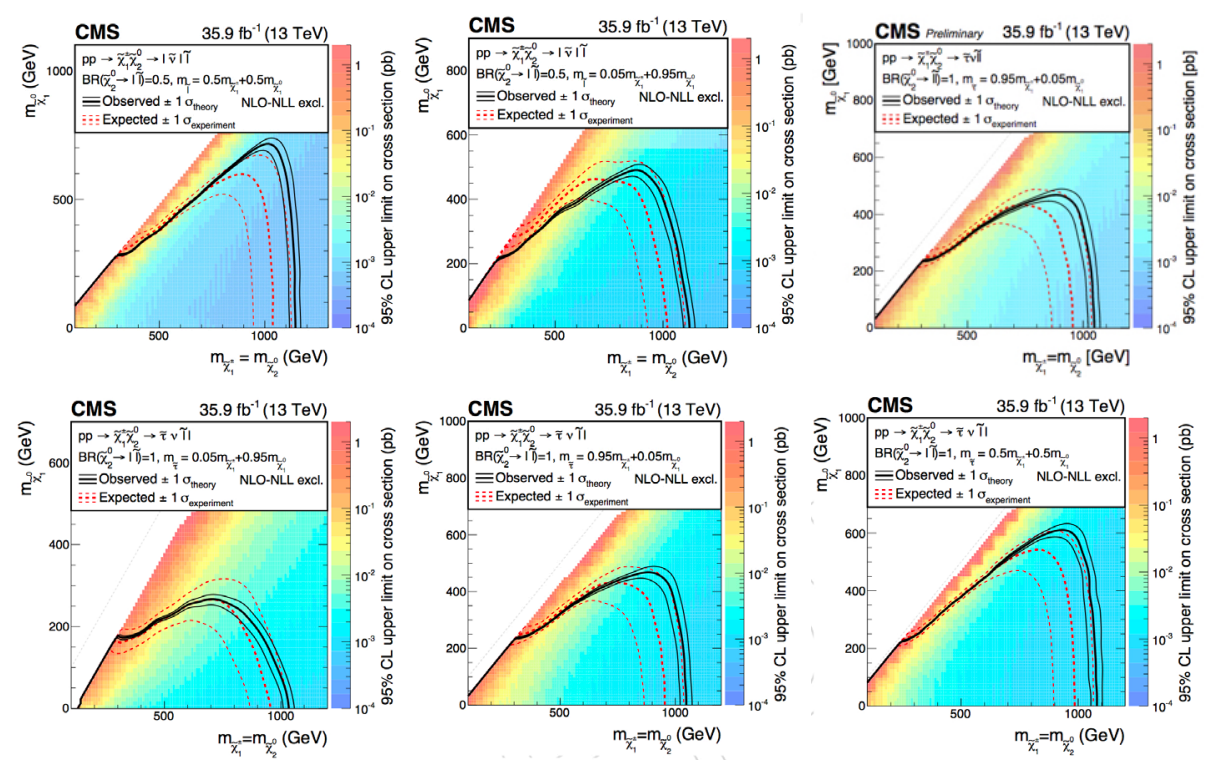

Figure 6: Some of the final results with the details of the model given in the legend of the plot. The full collection of results can be seen in the reference of the analysis [12]. 


\section{References}

[1] S. P. Martin Adv. Ser. Direct. High Energy Phys. 21 (2010) 1 [Adv. Ser. Direct. High Energy Phys. 18 (1998) 1] doi:10.1142/9789812839657_0001, 10.1142/9789814307505_0001 [hep$\mathrm{ph} / 9709356]$.

[2] J. Wess and B. Zumino, Nucl. Phys. B 70 (1974) 39. doi:10.1016/0550-3213(74)90355-1

[3] S. Chatrchyan et al. [CMS Collaboration], JINST 3 (2008) S08004. doi:10.1088/17480221/3/08/S08004

[4] J. Abdallah et al., Phys. Dark Univ. 9-10 (2015) 8 doi:10.1016/j.dark.2015.08.001 [arXiv:1506.03116 [hep-ph]].

[5] N. Arkani-Hamed, P. Schuster, N. Toro, J. Thaler, L. T. Wang, B. Knuteson and S. Mrenna, hepph/0703088 [HEP-PH].

[6] J. Alwall, P. Schuster and N. Toro, Phys. Rev. D 79 (2009) 075020 doi:10.1103/PhysRevD.79.075020 [arXiv:0810.3921 [hep-ph]].

[7] J. Alwall, M. P. Le, M. Lisanti and J. G. Wacker, Phys. Rev. D 79 (2009) 015005 doi:10.1103/PhysRevD.79.015005 [arXiv:0809.3264 [hep-ph]].

[8] D. Alves et al. [LHC New Physics Working Group], J. Phys. G 39 (2012) 105005 doi:10.1088/0954-3899/39/10/105005 [arXiv:1105.2838 [hep-ph]].

[9] https://twiki.cern.ch/twiki/bin/view/CMSPublic/PhysicsResultsSUS

[10] A. M. Sirunyan et al. [CMS Collaboration], Phys. Rev. D 96 (2017) no.3, 032003 doi:10.1103/PhysRevD.96.032003 [arXiv:1704.07781 [hep-ex]].

[11] A. M. Sirunyan et al. [CMS Collaboration], JHEP $\mathbf{1 7 1 0}$ (2017) 019 doi:10.1007/JHEP10(2017)019 [arXiv:1706.04402 [hep-ex]].

[12] A. M. Sirunyan et al. [CMS Collaboration], arXiv:1709.05406 [hep-ex]. 